\title{
Florence Nightingale y la profesionalización de la enfermería ${ }^{1}$
}

Florence Nightingale and the professionalization of nursing

\author{
Cristina Rodríguez Pastor \\ Facultad de Ciencias de la Educación \\ Universidad de Cádiz \\ cristina.rodriguez@uca.es
}

\section{Resumen}

En un momento de crisis epidemiológica sin precedentes en el que el papel del personal sanitario resulta clave, este artículo analiza la figura de Florence Nightingale como impulsora de la profesionalización de la enfermería y defensora de la educación de la mujer. Intentaremos explorar las claves de su éxito en la reforma de la profesión, así como algunos de los obstáculos a los que se enfrentó como consecuencia de las restricciones sociales. Al mismo tiempo, para entender mejor cómo este personaje se convirtió en icono de la cultura victoriana, revisaremos ejemplos concretos de su reflejo en la novela del siglo XIX.

Palabras clave: Enfermera, Profesionalización, Educación, División de roles.

\begin{abstract}
At a time when an unprecedented sanitary crisis has distinctively shown the key role of the health personnel, the present article intends to analyse the figure of Florence Nightingale as a precursor of the professionalization of nursing and early advocate of female education. We will try to explore the keys to her success in reforming the profession as well as some of the obstacles she faced because of the social restrictions of her time. Similarly, in order to provide a better insight into her transformation into a cultural icon, we will go through some specific examples of her representation in the Victorian novel.
\end{abstract}

Keywords: Nurse, Professionalization, Education, Division of roles.

${ }^{1}$ Recibido: 29/12/2020 Evaluado: 04/01/2021 Aceptado: 02/04/2021 


\section{Introducción}

A punto de finalizar 2020, declarado Año Internacional del Personal de Enfermería y de Partería por la Organización Mundial de la Salud en conmemoración del bicentenario del nacimiento de Florence Nightingale, este artículo pretende recordar el papel de esta defensora de la educación femenina, décadas antes de que el movimiento sufragista tomara impulso.

La insistencia tenaz de Nightingale en determinadas medidas como la importancia de lavarse las manos o de procurar una buena ventilación para prevenir contagios adquiere un significado determinante en la actualidad y nos permite entender la dimensión de la labor de este personaje.

En primer lugar, nos centraremos en su vida, destacando algunas experiencias cruciales que marcaron su trayectoria y descubriendo facetas generalmente menos conocidas. A continuación, comprobaremos de qué manera Nightingale intervino para lograr la regulación de la profesión de enfermera y cómo la ficción le sirvió de aliada en esta empresa. Seguidamente, analizaremos los factores que actuaron a favor del auge de la enfermera profesional y las dificultades que encontró a causa de los prejuicios sociales. Para terminar, reflexionaremos sobre la contribución de este icono de la cultura victoriana a la educación femenina y a su lucha por ocupar un lugar digno y respetado en la esfera pública.

\section{Apuntes biográficos sobre una mujer valiente}

Hija de una familia muy bien acomodada, la educación de la joven Nightingale, bajo la tutela de su padre, se centró en materias como las lenguas modernas y clásicas, la historia, las matemáticas, la gramática, la literatura inglesa y la música (McDonald, 2002).

Su inquietud por las causas sociales despierta en ella su vocación como enfermera, que se empeña en perseguir en contra de la voluntad de su familia, en especial de su madre y su hermana, quienes consideran esta profesión inadecuada para una mujer de su posición social. Hasta entonces, la ocupación de enfermera había sido propia de mujeres sin educación y de clase trabajadora y típicamente asociada a la propensión a la bebida y al lenguaje vulgar (McDonald, 2002). Nightingale aprovechaba sus viajes para visitar hospitales y entrevistarse con expertos médicos y enfermeras que, en algunos países, llevaban asentadas desde mediados de los años 40 en forma de hermandades religiosas de enfermeras (Bynum, 1994).

En 1850 consiguió convencer a su familia para permitirle realizar una estancia de formación en una escuela luterana para enfermeras en Alemania. Tan solo tres años más tarde, fue nombrada superintendente de un hospital londinense en el que realizó múltiples reformas, centrándose en particular en el papel de las enfermeras como elemento clave para el buen funcionamiento del hospital.

Durante la Guerra de Crimea en 1854, el Secretario de Guerra británico, Sidney Herbert, familiarizado con su labor, decidió enviarla a un hospital de campaña turco al mando de un equipo de 38 enfermeras, que habían sido formadas por ella misma, para prestar sus servicios. 
Cuando llegan al hospital de Scutari, la situación que se encuentran es crítica: a la falta de equipamiento básico médico, se sumaba la carencia de alimentos y un personal completamente desbordado. Los soldados heridos eran trasladados desde los campos de batalla en Crimea hasta Scutari en barco. El viaje duraba días y muchos morían por el camino. Los barracones que el gobierno turco había cedido a los británicos para levantar su hospital de campaña estaban construidos sobre un enorme alcantarillado. Los heridos se encontraban hacinados y sin apenas ventilación (McDonald, 2002). La labor de Nightingale y sus enfermeras para conseguir mejorar las condiciones sanitarias de Scutari y otros hospitales del frente fue monumental, más aún teniendo en cuenta la oposición tanto por parte de los propios médicos de los hospitales como por parte de algunos miembros de la Oficina de Guerra en Londres. Como explica Brasseur (2005): "This resistance had as much to do with the fact that she was a woman in a supervisory role as it did the nature of her position, which was seen as usurping military and medical hierarchy" (p.163).

La prensa se encargó de cubrir su experiencia durante los dos años que permaneció allí. La Guerra de Crimea fue la primera que contó con la presencia de corresponsales de guerra, incluyendo fotógrafos y artistas (McDonald, 2002). A la vuelta de Turquía, Nightingale se había convertido ya en una heroína. Su hábito de realizar su guardia nocturna en los hospitales de campaña alumbrándose con una lámpara de mano le valió el apodo de "Lady with the Lamp".

A pesar de haber contribuido a reducir el número de muertes en el hospital de Scutari, la comprobación de los datos concretos de mortalidad durante los meses posteriores a su regreso aumentó su preocupación. Era necesario hacer ver a las autoridades que gran parte de esas muertes podían evitarse, ya que se debían a enfermedades contraídas en los propios hospitales, no a heridas de guerra. De este modo, en 1856 Nightingale convence al gobierno para crear una Comisión Real con el fin de investigar las condiciones sanitarias del Ejército Británico en Oriente. De este modo, durante tres años, llevó a cabo un exhaustivo trabajo estadístico para analizar y demostrar las causas de los índices de mortalidad en los hospitales de guerra. Para esta tarea contó con la colaboración del estadístico y epidemiólogo William Farr (McDonald, 2002). Asimismo, realizó entrevistas a los médicos y los trabajadores de los propios hospitales, funcionarios militares, etc. con el fin de recoger sus observaciones (Brasseur, 2005).

Toda esta investigación constituyó la base científica sobre la que se apoyaban sus informes destinados a asesorar al gobierno británico sobre cómo prevenir las muertes por contagio y cómo mejorar las condiciones sanitarias en los hospitales y los destacamentos militares. Con el propósito de representar los datos de manera visual y facilitar su comprensión a lectores no necesariamente familiarizados con el lenguaje estadístico se valió del diseño de gráficos, entre los que destacó la creación de lo que ha venido a conocerse como el Diagrama de la Rosa (que en Matemáticas se conoce como Diagrama del área polar). Un ejemplo de uno de estos diagramas podemos verlo en la Imagen 1: 


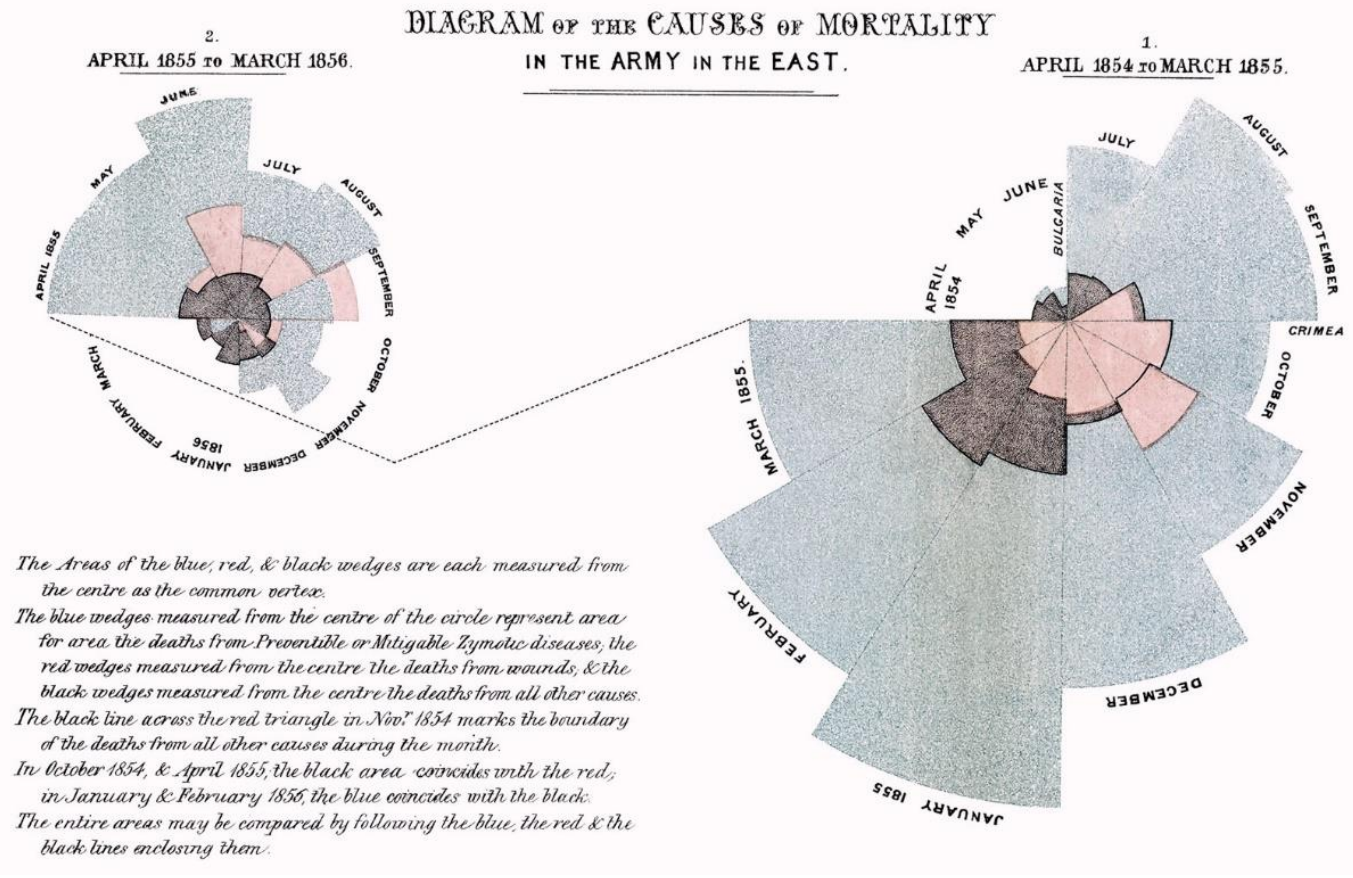

Imagen 1. Detalle del "Diagram of the Causes of Mortality in the Army in the East" de Florence Nightingale (1858). Fuente: www.vanderbilt.edu

En la leyenda inferior izquierda, Nightigale indica: "Las áreas en azul, rojo y negro están cada una medida desde el centro como vértice común. Las áreas azules medidas desde el centro del círculo representan área por área las muertes por enfermedades cimóticas, desde prevenibles a mitigables. Las áreas rojas medidas desde el centro, las muertes por heridas y las áreas negras medidas desde el centro, las muertes por el resto de causas. La línea negra que atraviesa el triángulo rojo en noviembre de 1854 marca el límite de las muertes por el resto de causas durante el mes. En octubre de 1854 y abril de 1855, el área negra coincide con la roja; en enero y febrero de 1856, la azul coincide con la negra. Las áreas completas se pueden comparar siguiendo las líneas azul, roja y negra que las contienen" (Traducción propia). Como señala Brasseur (2005), basta un simple vistazo al diagrama para comprender que el tifus, el cólera y la disentería causaban más bajas entre los soldados que los rusos.

Estos gráficos fueron incorporados como anexos a sus documentos. Además de remitir sus informes a la Oficina de Guerra, Nightingale publicó muchos de ellos en una versión más simplificada para el público en general, de ahí su interés por captar la atención del lector y hacer comprensibles los datos de un solo golpe de vista. Como Small (1998) explica, aunque obviamente Nightingale no fue la primera persona en emplear gráficos para reflejar datos estadísticos, probablemente fue de las primeras en usarlos para convencer de la necesidad de adoptar medidas urgentes para mejorar las condiciones de higiene en los hospitales.

Más adelante, en 1858, lideraría una segunda Comisión Real para tratar la situación del ejército en la India. A pesar de dedicar cinco años de su vida a investigar sobre las condiciones de vida en este país, nunca llegó a poder visitarlo por motivos de salud. 
Fue la primera mujer en ser nombrada miembro de la Royal Statistical Society en 1858, así como en obtener, en 1910, la distinción de la Orden del Mérito de manos de la propia Reina Victoria.

Florence Nightingale pasó la mitad de su vida en cama (más de 50 años) sin apenas poder recibir visitas por una enfermedad derivada de la fiebre que contrajo en Crimea, pero su invalidez no le impidió seguir trabajando incansablemente.

Su preocupación por la reforma de la sociedad le llevó a escribir una de las novelas más representativas del feminismo: Cassandra, escrita en 1854 y publicada dentro de su ensayo Suggestions for Thought to the Searchers after Truth Among the Artisans of England (1860). En esta novela la autora realiza un ataque frontal al rol pasivo de la mujer en la sociedad de la época, que le negaba el derecho a la educación superior y a la participación activa en la vida pública, advirtiendo sobre las consecuencias de esta inactividad en su salud mental.

El éxito de sus reformas hospitalarias radicó en la importancia concedida a una cuidadosa planificación, al buen funcionamiento y limpieza de las instalaciones, la higiene de los pacientes, la calidad de su alimentación, así como la disciplina y la profesionalidad de sus enfermeras. De este modo, la profesionalización de la medicina y la mejora de las condiciones de los hospitales coincidieron con la aparición de la figura de la enfermera profesional.

\section{Su papel en la profesionalización de la enfermería: The Nightingale Nurses}

Con las donaciones recibidas durante su estancia en Turquía se creó un fondo público que permitió la creación en 1860 de la primera escuela secular para la formación de enfermeras en el Hospital de St. Thomas de Londres: The Nightingale Training School for Nurses. Actualmente esta escuela forma parte del King's College.

El nuevo modelo de enfermera se situaba en profundo contraste con el modelo anterior, representado por la enfermera borrachina, ignorante, vieja y un tanto vaga popularizada en muchas novelas de la primera mitad de siglo, como fue el caso de la inolvidable Sairey Gamp en Martin Chuzzlewit (1843) de Dickens. Como Poovey (1995) afirma, el interés por la profesionalización de la enfermera se situaba en sintonía con las ansiedades de la sociedad, y de la medicina en concreto, por las condiciones de vida de los pobres y sus consecuencias:

The overcrowded and unsavory urban conditions made visible by the cholera epidemic of the 1840s was instrumental in drawing the attention of a worried middle class to the role of sanitation in the health and morality of the poor; this, along with an increased emphasis within the medical establishment on the importance of monitoring the patient's vital signs, mandated an attendant who was moral, observant, obedient, and capable of superintending sanitation. (p.174)

Nightingale fue también autora de un importante manual sobre enfermería que sigue editándose en la actualidad, Notes on Nursing (1860) Este manual no estaba solo destinado a las enfermeras que trabajaban en los hospitales o en las casas, sino a las mujeres en general,

No 9, 2021. Página| 273 
dando consejos básicos relacionados con la ventilación, la limpieza en las casas, la alimentación y los cuidados del enfermo, etc. En este sentido, se trata de una obra divulgativa: "...women, and the best women, are wofully deficient in sanitary knowledge; although it is to women that we must look, first and last, for its application, as far as household hygiene is concerned" (Nightingale, 1860b, p.13). Llaman la atención, sin duda, advertencias que hoy en día resuenan constantemente en nuestros oídos: "Every nurse ought to be careful to wash her hands very frequently during the day. If her face too, so much the better" (p.58). Sin embargo, en el último capítulo del manual (XIII Observation of the sick), su pautas para la formación de las enfermeras profesionales son claras:

The most important practical lesson that can be given to nurses is to teach them what to observe- how to observe- what symptoms indicate improvement- what the reverse - which are of importance — which are of none - which are the evidence of neglect - and of what kind of neglect. All this is what ought to make part, and an, essential part, of the training of every nurse. At present how few there are, either professional or unprofessional, who really know at all whether any sick person they may be with is better or worse. (p.65)

Del mismo modo, insistía en el valor del savoir faire frente a un conocimiento puramente conceptual: "practical manual nursing ... is impossible to learn it from any book ... it can only be thoroughly learnt in the wards of a hospital" (pp. 76-77).

Fue una gran defensora de la necesidad de promover la educación permanente de las enfermeras, al tratarse de una práctica que está en continua evolución (McDonald, 2002). En las cartas que escribía personalmente a sus enfermeras al final de cada año les recordaba que el progreso que hacían cada año en la escuela no era nada comparado con el que debían alcanzar cada año una vez terminada su formación (Nightingale, 1914).

\section{La figura de la enfermera en la novela victoriana}

En multitud de novelas de la época encontramos a mujeres ejerciendo de enfermeras bien como profesionales o simplemente haciéndose cargo del cuidado de familiares o amigos enfermos como parte de su rol doméstico. Ejemplos de estas últimas los encontramos en personajes como la propia Dorrit en Little Dorrit (Dickens, 1857) o Mrs. Pryor en Shirley (1849) de Charlotte Brontë. Su reflejo en la literatura constituye a menudo la personificación de valores asociados a la feminidad tales como la sensibilidad, la discreción y el sacrificio por los demás, de hecho, su función representa una extensión natural de su capacidad maternal. La propia Nightingale destacaba el vínculo entre la función doméstica y el papel de enfermera: "Every woman ... in England has, at one time or another of her life, charge of the personal health of somebody, whether child or invalid - in other words, every woman is a nurse" (Nightingale, 1860b, p.V). Para la cultura victoriana, la enfermera y el ángel del hogar eran roles intercambiables.

En cuanto a la enfermera profesional, un perfecto ejemplo lo encontramos en Hospital Sketches (1863) de Louisa May Alcott, novela casi autobiográfica donde la autora recreaba sus propias experiencias trabajando como enfermera para los soldados de la guerra civil

No 9, 2021. Página | 274 
americana. Su protagonista, Miss Tribulation Periwinkle, en las antípodas del personaje de Sairey Gamp mencionado antes, es el alter ego de Nightingale. La novela destaca la función de la enfermera como experta en la interpretación del lenguaje corporal del paciente: “... the silent eloquence of those long lines of faces ..." (p. 39).

Mientras que el interés del médico se centra obsesivamente en la materialidad del cuerpo del enfermo: "Dr. P. ... had acquired a somewhat trying habit of regarding a man and his wound as separate institutions ..." (p.91), la enfermera percibe la ruptura de la unidad del cuerpo y la mente del paciente. A través de la lectura de su cuerpo intenta reconstruir su significado emocional: "A slight wound in the knee brought him there; but his mind had suffered more than his body" (p. 45). Su capacidad para la empatía resulta, en cierto modo, sanadora.

En cualquier caso, frente al distanciamiento del médico, la figura de la enfermera en la literatura interviene en el conflicto de la simpatía hacia las emociones del paciente. Su labor complementa a la del médico ya que dispone de forma natural de las herramientas de las que los médicos supuestamente carecen o no pueden permitirse mostrar. De este modo, la medicina puede beneficiarse del trabajo de la enfermera sin poner en peligro la objetividad de su práctica.

\section{Claves del éxito de Nightingale y desafíos planteados}

Como se puede anticipar, la regulación de la profesión de enfermera no fue fácil en la época, dado que se trataba de un paso firme a favor de la participación de la mujer en la vida pública, alejada de la esfera doméstica a la que supuestamente pertenecía. Entre los factores que facilitaron esta transición podemos identificar:

- La coincidencia, como señalábamos antes, con la preocupación de la sociedad de la época respecto a las condiciones sanitarias, no solo en los hospitales, sino en las ciudades en general.

- La creación de un modelo de enfermera que estaba sustentado en los valores morales de la época y reforzado por la propia literatura.

- Las garantías de subordinación al médico, aspecto crucial para suavizar las susceptibilidades de la profesión médica respecto a las aspiraciones feministas de lucha por una educación superior. Como afirma McDonald (2002): “The nurses themselves, at Nightingale's insistence, worked solely under the direction of the doctors, the only way to get the doctors' co-operation" (p.29). De este modo, en el hospital se reproducía la división de esferas que la perspectiva de Nightingale traducía así: el medico prescribía medicinas que la enfermera administraba obedientemente al enfermo con total atención a sus instrucciones; por su parte, ella le aportaba observaciones de hechos, en lugar de opiniones, sobre el estado del paciente. Su ámbito de acción independiente consistía en favorecer un entorno cómodo y saludable a su paciente (Swenson, 2005).

- Las pésimas condiciones de trabajo: horarios abusivos, bajos salarios, sobreesfuerzo físico, etc. presentaban esta profesión como una actividad vocacional que no perjudicaría económicamente a la comunidad médica (Finkelstein, 1995).

Aun así, los colegios de médicos contemplaban con preocupación la profesionalización de las enfermeras. De hecho, la presencia de la enfermera profesional en los hospitales no estuvo 
exenta de graves tensiones derivadas de las relaciones de poder entre el médico y la enfermera y la propiedad de las relaciones entre ambos (Poovey, 1995). La irritabilidad hacia el nuevo modelo de enfermera a menudo surgía de sentimientos de inferioridad entre los propios médicos debido a su alto nivel de formación y conocimiento (especialmente en comparación con las antiguas enfermeras), que las llevaba en ocasiones a cuestionar algunas de las prácticas en los hospitales y a ser acusadas de insubordinación (Young, 2008).

Por tanto, el nuevo modelo de enfermera debía construirse en contraposición a los otros modelos que defendía no ser: "The image of the nurse had to be freed not only from the taint of its lower-class origin, but also from the contemporary specter of those "strong-minded women" who aspired to be doctors" (Poovey, 1995, p.173). Asegurar la separación de estos dos modelos al tiempo que se cultivaba la identificación de su actividad como una prolongación de su función doméstica seguía siendo problemático ya que cuestionaba la necesidad de proporcionarles un salario.

De este modo, el camino hacia la profesionalización de las enfermeras en la época victoriana no fue fácil. Los recelos hacia la incorporación de enfermeras profesionales a los hospitales reflejan la difícil intersección entre clase social y género en la sociedad inglesa de la segunda mitad del siglo XIX.

\section{Conclusión}

Como hemos podido comprobar, Florence Nightingale fue un personaje incómodo para la profesión médica: su continuo recordatorio de la necesidad de contar con enfermeras bien formadas y de proporcionarles un papel activo para mejorar las condiciones sanitarias de los enfermos suponía una alteración del statu quo en la sociedad de la época.

Su esfuerzo, sin embargo, no se limitó a la regulación de la profesión de enfermera, sino que acometió toda una campaña por la reforma sanitaria. Concretamente, intentaba demostrar que es posible reducir los niveles de incidencia de muchas enfermedades y aliviar, por tanto, la presión en los hospitales simplemente atendiendo a cuestiones básicas de higiene y salud pública. De nuevo, batallas que continuamos librando en la actualidad.

Con el paso de los años, se ha ido teniendo acceso a multitud de documentos que nos han permitido descubrir la profundidad de la huella de este personaje (diarios personales, cartas, informes, gráficos, estudios preliminares, entrevistas, actas de sus negociaciones con la Oficina de Guerra, etc.) y que dan testimonio de toda una vida dedicada al trabajo. A su faceta de enfermera añadimos así la de matemática, estadística, investigadora, negociadora, escritora y divulgadora, todas ellas caras de un prisma que dista mucho de la imagen de sencillez que reflejan sus escasas fotografías en las que posa serena rodeada de sus enfermeras.

\section{Referencias}

Alcott, L.M. (1993) Hospital Sketches. Bedford: Applewood Books. (Libro original publicado en 1863). 
Brasseur, L. (2005) Florence Nightingale's Visual Rhetoric in the Rose Diagrams, Technical Communication Quarterly, 14:2, pp. 161-182, DOI: 10.1207/s15427625tcq1402_3

Brontë, C. (1998) Shirley. Oxford: O.U.P. (Libro original publicado en1849).

Bynum, W. F. (1994) Science and the Practice of Medicine in the Nineteenth Century. Cambridge: Cambridge University Press.

Dickens, C. (2003) Little Dorrit. Londres: Penguin. (Libro original publicado en 1857).

Dickens, C. (2009) Martin Chuzzlewit. Oxford: OUP. (Libro original publicado en 1843).

Finkelstein, D. (1995). A Woman Hater and Women Healers: John Blackwood, Charles Reade, and the Victorian Women's Medical Movement. Victorian Periodicals Review 28.4, pp. 330-352.

McDonald, L. (Ed.) (2002). Collected Works of Florence Nightingale: An Introduction to her Life and Family. Waterloo: Wilfrid Laurier University Press.

Nightingale, F. (1914). Florence Nightingale to her Nurses. London: McMillan [Versión Kindle].

Nightingale, F. (1993) Suggestions for Thought to the Searchers after Truth Among the Artisans of England (Libro original publicado en 1860a). Nueva York: NYU Press.

Nightingale, F., \& Biology (Ed.) (1992). Notes on Nursing: What it is and What it is not. (Libro original publicado en 1860b) ProQuest Ebook Central. Recuperado de https://cutt.ly/1zZHnPa

Nightingale, F. (1858) "Diagram of the Causes of Mortality in the Army in the East". Recuperado de https://www.vanderbilt.edu

Poovey, M. (1995). Uneven Developments: The Ideological Work of Gender in MidVictorian England. Chicago: University of Chicago Press.

Small, H. (1998) "Florence Nightingale's Statistical Diagrams" en la Conferencia de Investigación Stats \& Lamps organizada por el Museo Florence Nightingale de St. Thomas' Hospital (Londres), 18 de marzo 1998.

Swenson, K. (2005) Medical Women and Victorian Fiction. Missouri: University of Missouri Press.

Young, A. (2008) 'Entirely a woman's question'? Class, Gender, and the Victorian Nurse. Journal of Victorian Culture 13.1, pp.18-41. 